\title{
THE FUNCTION OF THE QUINTUPLE INNERVATION OF A CRUSTACEAN MUSCLE
}

\author{
By A. VAN HARREVELD AND C. A. G. WIERSMA
}

The William G. Kerckhoff Laboratories of the Biological Sciences, California Institute of Technology, Pasadena, California

(Received 8 Fune 1938)

\section{(With Five Text-figures)}

\section{INTRODUCTION AND METHOD}

THE majority of the muscles in the chelipeds and walking legs of all crustaceans which have been investigated are innervated either by two or by three axons. These axons divide diplo- or triplotomically on the surface and within the muscle. The flexor of the carpopodite is in all cases an exception, since this muscle is innervated by five axons. These show a comparable mode of branching at the muscle: all of them dividing each time at the same place (quintuplotomic branching, van Harreveld, I939a). A sketch of such a division is presented in Fig. I. In the cheliped of Blepharipoda occidentalis two other exceptions are found; in addition to the quintuply innervated flexor of the carpopodite two quadruply innervated muscles are present.

In a triply innervated muscle two of the nerve fibres were proved to be motor axons, each eliciting a different type of mechanical response distinguished as "fast" and "slow" contraction. The third and thinnest fibre has a peripheral inhibiting function (van Harreveld \& Wiersma, 1937). In the doubly innervated muscles one motor and one inhibitory axon are present. At the time that the function of the triple innervation was described this was believed to be the typical innervation of crustacean muscle. After finding the quintuple innervation this conception had to be altered and an investigation of the function of the fibres in a quintuply innervated muscle became necessary.

Of the animals available the California rock lobster, Panulirus interruptus, was by far the most promising, since the axons innervating the flexor of the carpopodite are rather thick and the ischiopodite of the walking leg is relatively long. It is thus relatively easy to prepare the five axons over a distance of about $\mathrm{I} \mathrm{cm}$. and to stimulate them separately without danger of complications by current escape. In order to isolate the fibres in the ischiopodite it was necessary to amputate the leg in the coxopodite. Without special precautions such an amputation resulted in the death of the animal by bleeding, but by thermocauterizing the wound surface this bleeding could be checked. The shell was removed on the front side of the ischiopodite and adjoining parts of the meropodite; unless this is done with care, breaking 
at the line of autotomy results. The nerves are situated superficially at this side, covered by a membrane of strong connective tissue and some muscle fibres, which have to be removed before the actual isolation of nerve fibres can be started. Two main nerve bundles are present, a very thick and a thinner one both containing motor fibres. The five fibres for the flexor of the carpopodite run closely together in the thinner of the two bundles. These axons form, together with a few other thick fibres, a more or less distinctly separate part of this thinner bundle, which consists otherwise of a very large number of thin fibres. The actual isolation was performed by splitting the fibres in sea water under a binocular microscope. The fibres were stimulated separately with micromanipulated electrodes. Faradical stimulation, or stimulation with a thyratron device which generated rectangular currents of short
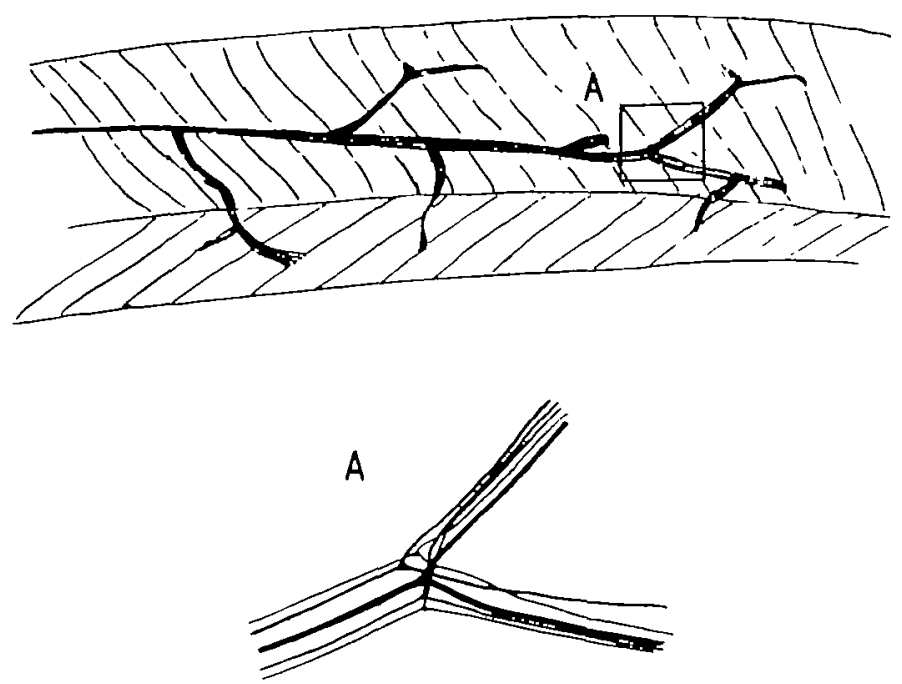

Fig. I. Sketch of the surface ramification of the five axons innervating the flexor muscle of the carpopodite of Panulirus. A shows a quintuplotomic branching enlarged.

duration at a wide variety of frequencies, was used. Action currents of the flexor muscle were recorded with a Matthews oscillograph; a grounded electrode was brought in direct contact with the muscle through a hole in the shell in the middle of the meropodite, the other electrode being placed on the tendon of the flexor muscle.

\section{MECHANICAL RESPONSES ON STIMULATION OF THE MOTOR AXONS}

After the bundle of thick fibres had been separated from the rest of the thinner main bundle described above, seven larger fibres which almost exclusively form this bundle could be isolated. Of these seven, two do not innervate the flexor of the carpopodite but, as stimulation of them showed, one is the common motor fibre for the extensor of the propodite and the abductor muscle and the other is the inhibiting fibre for the former of these two muscles. It could be shown in methyleneblue stained preparations that the remaining five larger fibres innervate the flexor 
of the carpopodite. Of these five, four are of a considerable diameter and thus easily distinguished from the fifth, which has only about one-third of the diameter of the thinnest of the other four. Faradic stimulation of any of the four thick fibres caused a contraction; stimulation of the fifth did not do so.

When the four contractions elicited by stimulation of the four motor axons with the same frequency were compared they showed differences in shape and speed, as illustrated in Fig. 2. In this preparation it is obvious that there is a considerable difference in the speeds of the contractions. These differences are not always as clear as in the instance given, since often two of the four contractions resemble each other closely. However, using various frequencies of stimulation, it

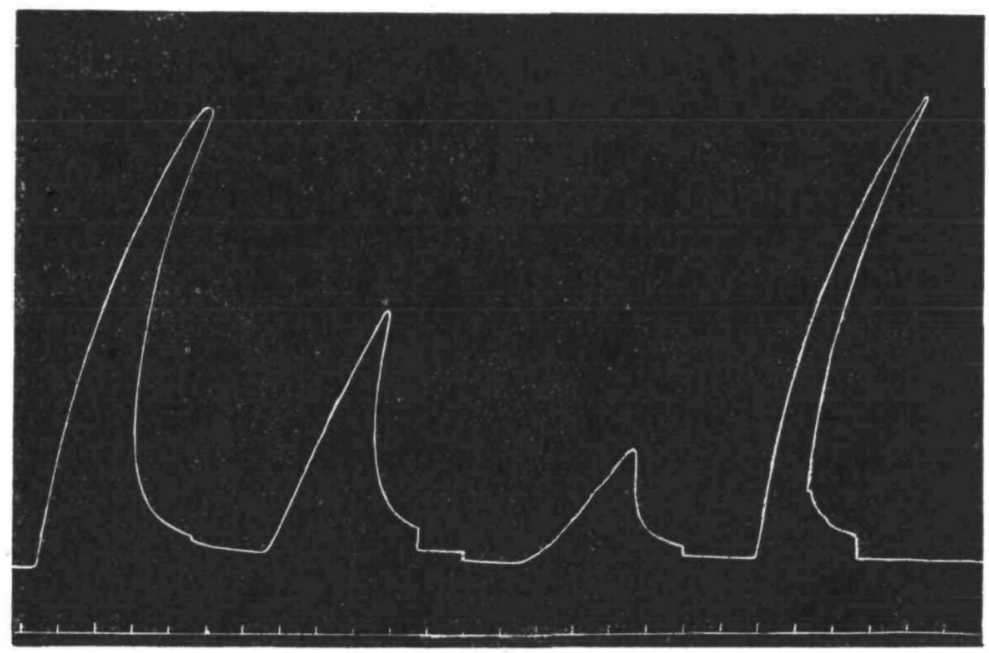

B

$\mathrm{C}$

D

A

Fig. 2. The four contractions elicited by stimulation of the four motor fibres at a frequency of 30 per sec. A, diameter of the axon $129 \mu$, quickest contraction; B, diameter of the axon $89 \mu$, 8econd quickest contraction; $\mathrm{C}$, diameter of the axon $91 \mu$, third quickest contraction; $\mathrm{D}$, diameter of the axon $122 \mu$, slowest contraction. Time I sec.

is always possible to select one at which a striking difference between any given pair of the contractions is obtained. There can thus be no doubt of the reality of the differences described, and it must be concluded that stimulation of each of the four motor fibres elicits a contraction of a specific character. As further evidence, each fibre has often a special minimum frequency which elicits a just visible mechanical response. For instance, in a certain preparation stimulation of one fibre still caused a contraction when stimulated at a frequency of 2 per sec.; for the other fibres this figure was respectively $4, I_{3}$ and 17 per sec. For any given fibre the actual minimum frequency varies in the course of an experiment, partly due to differences in facilitation and partly to ageing of the preparation. By facilitation a contraction may be obtained with a stimulation frequency at which no contraction resulted before; on the other hand, the minimum frequency increases 
with ageing. These factors make it impossible to use the minimum frequency determination as a method for identifying with certainty the different contractions.

The two motor axons of the adductor muscle in various marine and fresh-water crustaceans can be identified with certainty as the "fast" and the "slow" fibre by the relation of their diameters, which is on the average $I \cdot 4:$ I (van Harreveld \& Wiersma, 1936, I937; Wiersma \& van Harreveld, 1938a). Measurements of the diameters of the four motor fibres and comparison of these with the contractions obtained made it evident that in this case the speed of the contraction does not increase with the fibre thickness (Fig. 2). Nevertheless the diameter of a fibre can be used to a certain extent as an indicator of the type of contraction elicited by it. Usually two very thick and two thinner fibres are present; in such preparations one of the thicker fibres elicited the quickest contraction, the other the slowest, and the two thinner fibres caused the intermediate contractions. It was found that in these preparations the fibre causing the quickest contraction might be either somewhat thicker or thinner than the fibre eliciting the slowest contraction.

With the higher frequencies used the speed relations of the four contractions remain unchanged, but with low frequencies the quickest contraction often decreases relatively more than the others. This decrease can be so pronounced that the quickest contraction is not elicitable at a frequency which still gives a fair contraction when applied to the next quickest fibre. At these low frequencies (around ro per sec.) the other two fibres usually do not elicit a contraction.

Isotonic and isometric records of the four contractions show that the relaxation speed of the second fastest contraction is much slower than the others. This phenomenon was most clearly demonstrated when the frequencies with which the different fibres were stimulated were chosen in such a way that the resulting contractions reached, in about the same time of stimulation, the same height.

\section{THE MUSCLE ACTION CURRENTS ON STIMULATION OF THE FOUR MOTOR FIBRES}

The stimulation of the four motor fibres not only caused four different kinds of mechanical response but also four different types of muscle action current. One of these (type I) is outstandingly different from the three others, the action potentials being several times larger than those of any of the others. The muscle action current caused by the first nerve impulse is rather large in this type, but a very distinct increase of the spikes (facilitation) takes place in the course of a faradic stimulation. In type II of the action currents the spikes are much smaller, but still of a fair height. The action current caused by the first nerve impulse is very small, but the spikes grow quickly during the first moments of faradic stimulation. The spikes of the third and the fourth type of action current are very small. There seems to be no pronounced facilitation in either of them; this, however, is difficult to investigate because of the smallness of the spikes. The spikes of these two types reach sometimes the same height during faradic stimulation, but usually one is somewhat larger than the other. There is a typical difference in 
the shape of the action currents of these two types which, though hard to describe, is usually very distinct. The spikes of one type (type III) show a more abrupt fall after the peak has been reached than those of the other (type IV). In Table I the height of the action currents of the four types after I sec. of faradic stimulation is given.

Table I. Correlation between the type of action current, the height of the spike after I sec. of faradic stimulation in $\mathrm{mm}$. and the diameter of the axon in $\mu$ (between brackets)

\begin{tabular}{|c|c|c|c|c|c|}
\hline $\begin{array}{c}\text { Type of } \\
\text { action } \\
\text { current }\end{array}$ & \multicolumn{5}{|c|}{ Preparation no. } \\
\cline { 2 - 6 } & I & 2 & 3 & 4 & 5 \\
\hline I & $17 \cdot 5$ & 36 & 24 & 30 & 13 \\
& $(92)$ & $(140)$ & $(120)$ & $(136)$ & $(124)$ \\
II & $6 \cdot 2$ & $3 \cdot 5$ & $6 \cdot 0$ & $6 \cdot 0$ & $2 \cdot 7$ \\
& $(88)$ & $(88)$ & $(63)$ & $(76)$ & $(88)$ \\
III & $2 \cdot 0$ & 0.8 & $2 \cdot 9$ & $1 \cdot 0$ & $1 \cdot 4$ \\
& $(100)$ & & $(86)$ & $(90)$ & $(92)$ \\
IV & $0 \cdot 5$ & $(1 \cdot 1$ & $1 \cdot 5$ & $2 \cdot 2$ & 0.7 \\
& $(120)$ & $(108)$ & $(116)$ & $(133)$ & $(126)$ \\
\hline
\end{tabular}

In preparations in which the four fibres are divided into two thicker and two thinner ones, the types I and IV of action currents are elicited by stimulation of the thicker fibres, the types II and III by stimulation of the thinner ones.

In some experiments the four types of muscle action current were taken from different parts of the muscle; instead of two, three holes were made in the shell, thereby providing three possible ways of leading off the muscle action currents with the grounded electrode placed proximally. These experiments were performed to show that the structures responsible for the four types of action current are distributed equally throughout the muscle. In a certain preparation, for instance, in which the whole flexor muscle had a length of $40 \mathrm{~mm}$., one hole was made over the tendon, the second I I mm. and the third $23 \mathrm{~mm}$. proximal. The heights of the action currents of each type were different with the three ways of leading off, but the relation of the heights remained the same (Table II).

Table II. Relation of the heights of the four different types of action current led off in three ways, the height of the action current obtained with the second way of leading off being taken as unity

\begin{tabular}{|c|c|c|c|}
\hline $\begin{array}{c}\text { Type of } \\
\text { action } \\
\text { current }\end{array}$ & \multicolumn{3}{|c|}{ Way of leading off } \\
\cline { 2 - 4 } & I & 2 & 3 \\
\hline I & I.4 & I & I.8 \\
III & I.4 & I & I.7 \\
IV & $1 \cdot 4$ & I & I.9 \\
\hline
\end{tabular}


A

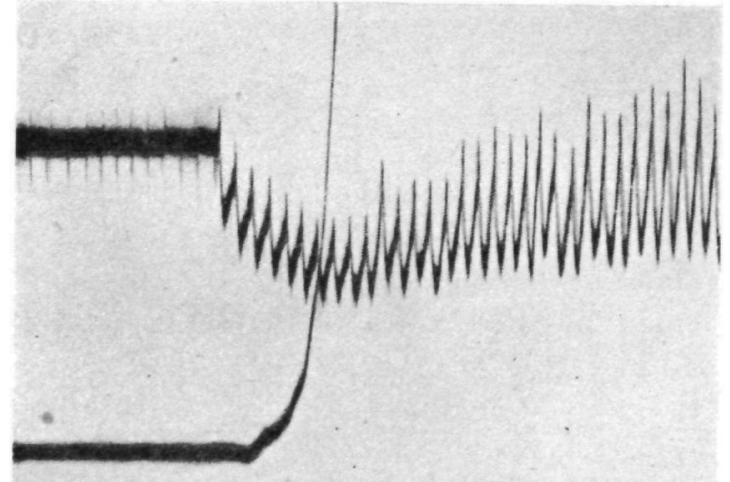

B

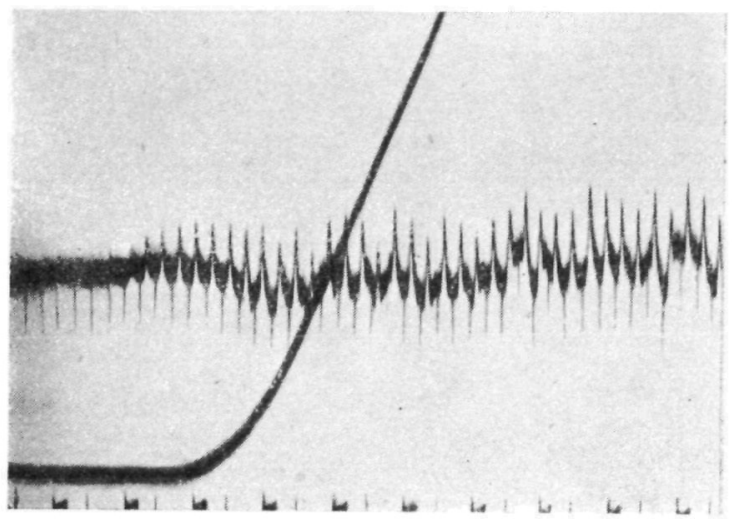

C

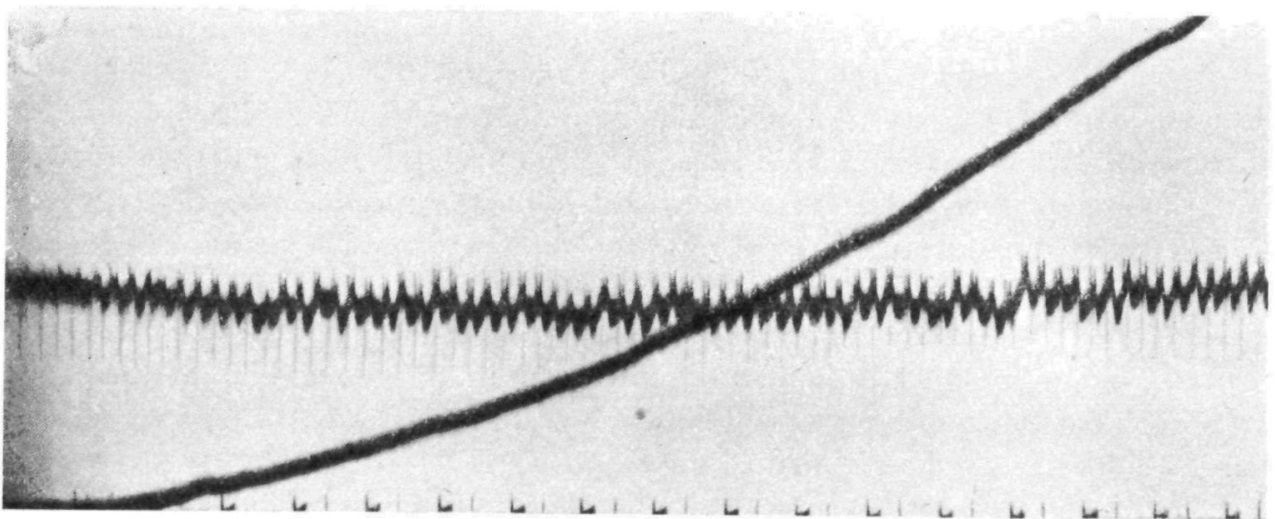

D

Fig. 3 . 


\section{Function of Quintuple Innervation of a Crustacean Muscle}

The relation between the four types of action currents and the four mechanical responses was studied by recording the electrogram and the mechanogram simultaneously. In preparations in which the four types of mechanical response showed the usual correlation between fibre size and contraction speed, as described above, it was found that the four types of electrograms were related to those contractions in a certain way. The fastest contraction, caused by the stimulation of a thick fibre, was accompanied by the large action currents of type I; the second fastest contraction, caused by the stimulation of one of the thin fibres, by the fairly large action currents of type II; the third fastest contraction, caused by the other thin fibre, by the action currents of type III; and the slowest contraction, caused by the stimulation of the second of the thick fibres, by the action currents of type IV. The correlation between the diameter of the motor fibres, the four types of the action currents and the speed of the mechanical response at the same frequency of stimulation is illustrated in Fig. 3 .

Stimulating with frequencies at which the contraction accompanied by the action currents of type $I$ is no longer visible, but at which the contraction with the action currents of type II is still pronounced (see above), a phenomenon described as the "paradox" can be observed (Wiersma \& van Harreveld, 1938b). The action currents of type $I$ are under these circumstances much higher than those of type II, but only the latter are accompanied by a visible contraction.

\section{THE MUTUAL INFLUENCE OF THE FOUR MOTOR FIBRES}

In preparations in which three of the motor fibres required at a certain point in the course of the experiment almost the same minimum frequency to elicit a visible contraction, all three together were stimulated on one pair of electrodes at this minimum frequency. The resulting contraction was never noticeably larger than that of the sum of the contractions obtained by stimulating the fibres separately. When the frequency of stimulation was made just too low for each of the fibres when stimulated separately no contraction was obtained by stimulating all three axons together. Thus no mutual influence can be demonstrated in this way.

The mutual influence was further investigated with a method which in the adductor of Cambarus clarkii resulted in the demonstration of a distinct influence of one kind of contraction on the other (Wiersma \& van Harreveld, 1939). In Panulirus one of the two thinnest fibres was stimulated for $3 \mathrm{sec}$. at such a frequency that a small contraction resulted. This stimulation was repeated with intervals of $2 \mathrm{~min}$. until the ensuing contractions were nearly constant in shape and height. Then such a stimulation was preceded by a 30 sec. faradic stimulation of one of

Fig. 3. The four action currents and contractions elicited by faradic stimulation of the four motor fibres. A, diameter of the fibre $124 \mu$; action currents of type I; contraction is the fastest. Notice the size of the first action current and the marked facilitation. B, diameter of the fibre $63 \mu$; action currents of type II; the contraction is the second quickest. The first action current is small, but there is rapid growth by facilitation. C, diameter of the fibre $86 \mu$; action currents of type III. D, diameter of the fibre II $6 \mu$; action currents of type IV. The contractions in C and D are almost equal in this preparation at this frequency. Time in to sec. 
the other fibres ending I $_{5} \mathrm{sec}$. before the test contraction was due. The two thick fibres are not very suitable as test fibres, since the fastest contraction is at low frequencies of stimulation rather irregular, whereas the minimum frequency for the slowest contraction varies too much during the course of the experiment. It was possible to study in one preparation the influence of three fibres on the contraction caused by one of the thin fibres, and afterwards to do the same using the other thin fibre as test fibre.

In a typical preparation a facilitating influence was found in every one of the six possible combinations. This facilitating influence was about equal for the faradic stimulation of each of the fibres, and the effect of heterofacilitation was always much less than that of a similar faradic stimulation of the test fibre itself (autofacilitation). In some preparations the effect of the faradic stimulation of the other fibres on the test contraction was not a facilitation but a depression, a result which has also been obtained in Cambarus.

\section{THE FUNCTION OF THE FIFTH FIBRE INNERVATING THE FLEXOR OF THE CARPOPODITE}

The diameter of the fifth fibre is much less than that of the four motor fibres, as is shown in Table III. It has been stated above that stimulation of this fibre never results in a contraction. When this axon is stimulated simultaneously with one of the motor fibres it causes a peripheral inhibition of the contraction (Fig. 4).

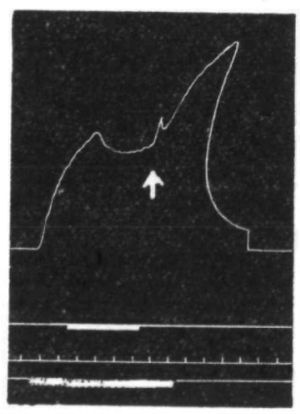

A

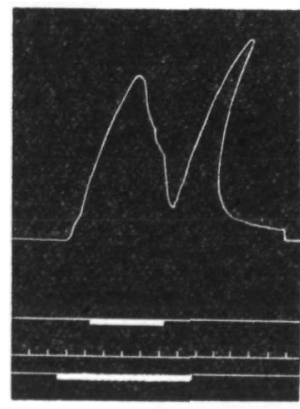

B

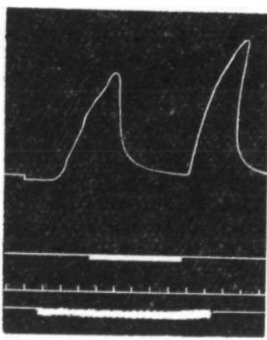

C

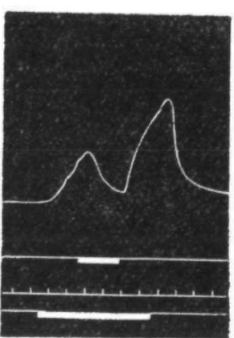

D

Fig. 4. Inhibition of the four contractions of the flexor of the carpopodite. Upper signal indicates stimulation of the inhibitor, lower signal stimulation of a motor fibre, both faradically. The contractions are arranged according to their speed. The diameter of the motor fibres is, in $A$, I $_{2} 6 \mu$; in $\mathrm{B}, 74 \mu$; in $\mathrm{C}, 8 \mathrm{I} \mu$, and in $\mathrm{D}$, $110 \mu$. The diameter of the inhibitor fibre is $32 \mu$. Notice that in $A$ inhibition is only partial and shows at the arrow a temporary escape. Time a sec.

Wedensky inhibition by current escape is certainly not responsible for this inhibition since injury of the fibre abolishes it. Of the contractions elicited by the stimulation of the four motor fibres the quickest one is the most difficult to inhibit. To suppress this contraction totally the frequency of the inhibiting stimulus must be higher than that applied to that motor fibre. For instance, when the inhibitory fibre was stimulated with a frequency of 45 per sec. no contraction resulted on a simultaneous 
stimulation of this motor fibre at a frequency of 20 per sec., but a small contraction was obtained with a frequency of 25 per sec. (incomplete inhibition). Complete inhibition of the contractions elicited by a faradic stimulation ( 45 per sec.) of the three other motor fibres is obtained when the inhibiting fibre is stimulated with about the same frequency. Decreasing the frequency of the inhibiting stimulus or increasing the frequency of the stimulus of the motor fibres makes inhibition incomplete; no significant differences were found in this respect between the latter three motor fibres.

Table III. Diameter of the inhibitory and the four motor fibres. The motor axons are arranged according to the speed of contraction (I being the fastest, etc.). The order in which the contractions are arranged presumably corresponds with the order of their types of action current, as given before.

\begin{tabular}{|l|r|r|r|r|r|}
\hline \multirow{3}{*}{ Axon } & \multicolumn{5}{|c|}{ Preparation } \\
\cline { 2 - 6 } & A & B & C & D & E \\
\hline Inhibitor & 24 & 35 & 34 & 32 & 34 \\
Motor I & 148 & 152 & 146 & 126 & II \\
Motor II & 108 & 118 & 94 & 74 & 108 \\
Motor III & 117 & 118 & 99 & 81 & II \\
Motor IV & 142 & 160 & 148 & 110 & 148 \\
\hline
\end{tabular}

The effect of inhibition on relaxation has been studied in different ways. Inhibition applied during the last part of a relaxation causes a slight increase in the speed of it in all four contractions. In another experiment the uninhibited relaxation was compared, on the one hand, with the relaxation caused by stimulating the inhibitory fibre simultaneously with the motor fibre, and on the other hand, with the relaxation obtained by starting the inhibition at the moment that the stimulation of the motor fibre was stopped. Pronounced differences were obtained in the relaxations of the slowly relaxing contraction (second quickest contraction). In this case both inhibition during and after stimulation of the motor fibre caused a quicker drop than the uninhibited relaxation (Fig. 5).

\section{THE ACCESSORY MUSCLE OF THE CARPOPODITE}

In all the decapod crustaceans investigated an accessory flexor muscle of the carpopodite has been observed which was described before in Cambarus (van Harreveld \& Wiersma, 1937). The muscle consists of two small muscle bellies connected by a long tendon, as in the mammalian digastric muscle. In methyleneblue preparations of Panulirus the distal belly was found to be doubly innervated. In the thick main nerve bundle both fibres innervating this belly have been isolated; stimulation of the thickest of the two caused a contraction in the muscle which was inhibited by a simultaneous stimulation of the thinnest. The motor fibre $(76 \mu)$ was somewhat thinner than the motor fibres of the main flexor muscle, the inhibiting fibre $(34 \mu)$ was of the same diameter as that of the main flexor. The separate and 
different type of innervation of this muscle indicates that it can contract independently from the main flexor muscle. The functional importance of this muscle is not clear.

In the course of the preparation of these two fibres for the accessory flexor muscle a number of other motor and inhibitory fibres were found in the thick and in the thin main bundles in the ischiopodite. It was interesting to isolate, stimulate and measure all these axons from one preparation. In the thick main bundle of

A

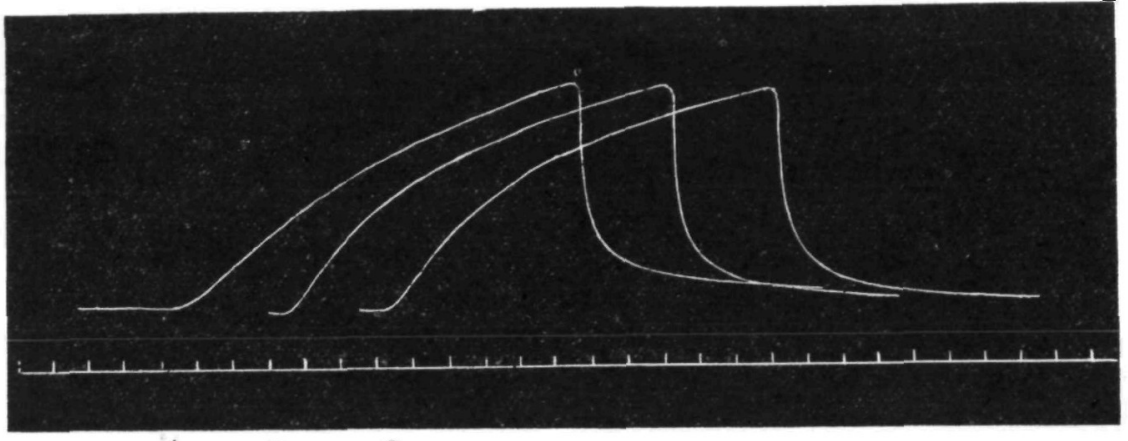

$1 \quad 2 \quad 3$

B

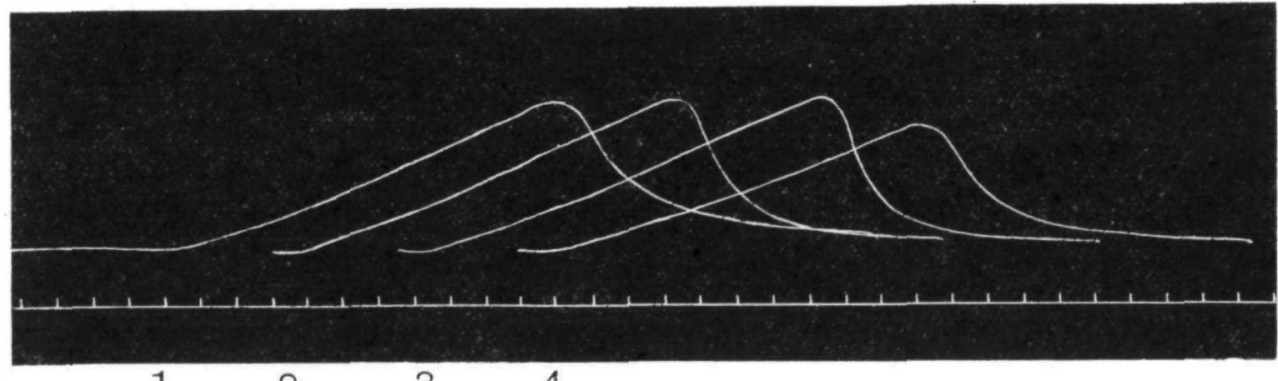

12

3

4

Fig. 5. The influence of inhibition on relaration. A, contractions of the III type of equal duration (10 sec.); frequency 20 per sec. A I, stimulation of the motor fibre alone; A 2, after ro sec. simultaneous stimulation of the inhibitor; $A_{3}$, after ro sec. stimulation of the motor fibre stopped, but that of inhibitor started. There is no marked difference in relaxation. B, contractions of II type 10 sec.; frequency 12 per sec., relaxation much slower than in $A_{\text {. }} B_{1}$ as $A_{1} ; B_{2}$ as $A_{2}$, inhibition simultaneously with excitation produces pronounced quicker relaxation than in $B_{1} ; B_{3}$ as $A_{3}$, relaxation is still quicker. $\mathrm{B}_{4}$, as $\mathrm{B}_{\mathrm{I}}$, shows again slow relaxation. Time $\mathrm{I}$ sec.

the preparation mentioned above the following fibres were obtained in addition to the two fibres for the accessory muscle: two motor fibres ( 118 and $108 \mu$ ) for the adductor of the dactylopodite, two motor axons (135 and $120 \mu)$ for the flexor of the propodite, the inhibitor fibre ( $108 \mu$ ) for this muscle, and the two motor axons ( 162 and $135 \mu$ ) for the extensor of the carpopodite. In the thin main bundle the seven motor and inhibitor fibres were isolated, namely the four motor axons ( $160,152,118$ and $118 \mu$ ) for the flexor of the carpopodite, the inhibiting fibre $\left(3^{6} \mu\right)$ for this muscle, the common motor fibre $(108 \mu)$ for the abductor of the 


\section{Function of Quintuple Innervation of a Crustacean Muscle}

dactylopodite and the extensor of the propodite, and the inhibiting fibre $(72 \mu)$ for this last muscle. It is likely that in this preparation all the motor fibres for the seven distal muscles of the walking leg were found, but only part of the inhibiting axons.

\section{DISCUSSION}

It has been possible to elicit four different kinds of contraction in the flexor muscle of the carpopodite, and the question arises whether these four contractions are due to the existence of four different sets of muscle fibres each innervated by one of the motor axons, or to two sets of muscle fibres with a double motor innervation, or to all the contractions occurring in the same set of fibres. The constancy of the ratios of the four action currents when led off from different parts of the flexor muscle proves that the structures in which the four kinds of contraction are elicited are evenly distributed in the muscle. In triply innervated muscles it has been proven that at least part of the muscle fibres can contract in two ways and receive branches of all three nerve fibres (Wiersma \& van Harreveld, $193^{8} b$; van Harreveld, $1939 b$ ), and it is very likely that the triplotomic branching observed in such muscles is by itself proof of the triple innervation of each muscle fibre. Correspondingly the quintuplotomic branching of the axons innervating the flexor muscle may be taken as an indication for the quintuple innervation of the muscle fibres. Physiological and anatomical evidence supports this. Since all the contractions can be inhibited by the same inhibitor it is certain that at least this nerve fibre is common to all muscle fibres. In silver stained preparations it has been possible to trace at least three different axons to the same muscle fibre (van Harreveld, 1939a), which excludes the possibility of four different sets of muscle fibres. The possibility of two sets of doubly motor innervated fibres is made unlikely by the experiments on the mutual influence of the four contractions. The amount of heterofacilitation was about the same in all combinations tried, which could hardly be expected if two sets of doubly motor innervated fibres were present, since in that case a much larger influence of the contraction in the same set of muscle fibres compared with that of the other set ought to be found. The same considerations exclude other much less likely possibilities, as for instance that one contraction is elicited in one set of muscle fibres and the three others in a second set.

If the four contractions of the flexor muscle occur in the same muscle fibres it may be asked which of them can be compared with the slow and the fast contraction of a doubly motor innervated muscle such as the adductor. In an attempt to answer this question action currents of the slow and the fast contraction have been led off from the adductor muscle in Panulirus during faradic stimulation. These action currents showed the same features as those previously obtained in the adductor of Cancer (Wiersma \& van Harreveld, 1938a), viz. the action currents of the fast contraction start with a rather small spike but are facilitated to a considerable height, those of the slow contraction are very small at the beginning of a stimulation but become larger than the first spike of the fast contraction. These features make them comparable with the action currents of the two fastest 
contractions (types I and II) of the quintuply innervated flexor of the carpopodite. There is, however, an important difference between the slow contraction of the adductor and the second quickest contraction of the flexor, since the relaxation of the former is not noticeably different from that of the fast contraction, while the second quickest contraction of the flexor shows the slow relaxation described.

The experiments presented in this paper are in agreement with the conception of the crustacean nerve-muscle system developed in previous papers (Wiersma $\&$ van Harreveld, $1938 b$; van Harreveld, $1939 b$ ). It has been found anatomically that on each crustacean muscle fibre a large number of nerve endings are present. In the case of a doubly motor innervated muscle these endings are formed by both motor fibres. A similar arrangement of any number of motor fibres is in principle possible as long as the endings of each fibre are well distributed over the whole surface of the muscle fibre, since this is the way in which the impulse is distributed over the crustacean muscle fibre. As a matter of fact, muscles have been found with a single, a double and a quadruple motor innervation, and it is likely that in Blepharipoda occidentalis the two quadruply innervated muscles that have been found in the cheliped are triply motor innervated.

The nerve muscle system of the crustaceans has developed along entirely different lines from that of the vertebrates. Though a very small number of motor fibres is present for each muscle, this nerve muscle system may be considered as well adapted to its function as that of the vertebrates. In the latter, contractions can be varied in two ways: by changes in the number of motor fibres in action or by changes in the frequency of the discharges in each of the motor fibres. Both mechanisms are also available in the crustaceans, but the first one to a much lesser extent. In addition each of the motor fibres of the crustaceans elicits a contraction with very marked characteristics which may be useful for special purposes. Since the characteristics of comparable contractions can. again differ from muscle to muscle there is the possibility that each muscle has a mechanism for exciting the contractile substance in accordance with its function. Of the more distal muscles in the leg, the flexor of the carpopodite is the most important for support of the body weight and it is of interest to note that this muscle is quintuply innervated in all the decapods examined. Since it was shown in a previous paper (Wiersma \& van Harreveld, $1938 b$ ) that fatigue is mainly situated in the intermediary processes between the nerve impulse and the contractile substance, the presence of two or more axons ending on the same muscle fibre would be a very efficient way to counteract such a fatigue.

\section{SUMMARY}

The functions of the five fibres innervating the flexor muscle of the carpopodite was investigated in Panulirus interruptus. The four thicker fibres were found to be motor axons, each eliciting a contraction with different characteristics. These four contractions were accompanied by four different types of action currents.

The thinnest fibre when stimulated simultaneously with any of the four motor fibres caused inhibition of the contraction. 
It is concluded that all four contractions take place in all the muscle fibres and that the conception of the mechanism of crustacean nerve muscle system developed before is enlarged to include the new results. The possible biological significance of the quintuple innervation is discussed.

\section{REFERENCES}

van Harreveld, A. (1939a). F. comp. Neurol. (in the Press).

$(1939 b)$. F. comp. Neurol. (in the Press).

van Harrevelo, A. \& Wimresma, C. A. G. (1936). F. Physiol. 88, 78.

(1937). F. exp. Biol. 14, 448.

Wiersma, C. A. G. \& VAN HarRevel.d, A. (1938a). 7. exp. Biol. 15, 18.

- (1938b). Physiol. Zool. 11, 75. 